\title{
Localization of late potentials using body surface mapping
}

\author{
Linnenbank A.C. ${ }^{1,2}$, van Dessel P.F.H.M. ${ }^{3}$, Potse M. $^{2}$, de Bakker J.M.T. ${ }^{4}$, van Hemel N.M. ${ }^{3}$ \\ ${ }^{1}$ Heart Lung Center Utrecht, The Netherlands, ${ }^{2}$ Academic Medical Center, Amsterdam, The Netherlands, \\ ${ }^{3}$ Antonius Hospital, Nieuwegein, The Netherlands, ${ }^{4}$ Interuniversity Cardiology Institute Netherlands
}

\section{Introduction}

Late potentials recorded at the body surface as defined by Simson [1] are assumed to reflect delayed activation in a myocardial substrate. However, in a substantial subset of patients with documented ventricular tachycardia (VT) no late potentials can be detected on the signal averaged ECG (SAECG). Also direct proof of the anatomic correlation between intracardial signals and the surface leads is still lacking. This study aimed to investigate the relation between simultaneously recorded late potentials at multiple sites on the body surface (SABSM) and late/diastolic activation within the heart utilizing a multi-lead endocardial electrode array. Several technical considerations should be accounted for when correlating intracardiac activity with late potentials detected with SABSM.

First, it is required that the $3 \mathrm{D}$ positions of the electrodes in the heart are known in order to make the spatial correlation. Second, it should be noted that the amplitude of late potentials in averaged signals in any lead will not only be influenced by the size and orientation of the underlying electrical wave front but also by the distance of the source to the electrode. The amplitude of a signal generated by a dipole will diminish rapidly with the distance between source and recording electrode. This means that irrespective of the location of the substrate in the heart the maximum amplitude of late potentials will usually be recorded in the precordial region. To avoid underestimation of the significance of late potentials in other chest areas, a correction for the effect of electrode-source distance is needed. Since the potential measured at the surface is a result of the potential distribution at all points in the heart and a transfer matrix, this transfer matrix should be estimated. The transfer matrix could be computed from volume conductance theory and information derived from MR images. For practical purposes, however, a measure that can be derived from the data itself is preferable.

\section{Methods}

Sixteen patients admitted to the Antonius hospital between November 1997 and May 1998 were studied. All patients had documented VT. Basic patient characteristics are given in Table 1.

In this study we recorded 65 leads at the body surface (62 leads according to the Amsterdam lead system plus three extremities) simultaneously with 61 endocardial leads from a Constellation ${ }^{\mathrm{TM}}$ basket catheter (Boston Scientific Inc.). Biplane X-ray images were acquired and digitized to obtain 3D positions of all endocardial electrodes.
Standard $\mathrm{X}, \mathrm{Y}$ and $\mathrm{Z}$ leads were reconstructed from a selection of 3 electrode pairs from the 65 body surface electrodes that matched the positions of the Frank leads best. The standard SAECG was obtained from these $\mathrm{X}, \mathrm{Y}$ and $\mathrm{Z}$ leads to compare SABSM with the signal averaging method that is currently applied clinically.

As a time reference for all surface leads the $\mathrm{J}$ point in the unfiltered QRS was chosen. The energy in the $50 \mathrm{~ms}$ following this point was integrated and plotted as a body surface map.

Because the precordial region is closest to the heart, the largest energy contributions from late diastolic potentials will in general be found in this region.

During left ventricular depolarization every cell generates a small current, the summation of which results in the QRS complex at the body surface. It would seem that for every point at the surface, the average energy during the QRS complex could be used as an estimate of the distance effect matrix, because it depends on the average distance of all points in the heart to the recording electrode. This assumption, however, should be treated with caution since most dipoles during cardiac activation point more or less in the same direction. So the energy content at positions at the body surface that are perpendicular to the heart axes, will then be underestimated. For a better estimate of the distance effect we need a collection of dipoles. Pacing at multiple sites in the ventricle could be employed, but that is

\begin{tabular}{|l|l|}
\hline Age (years) & $67.5 \pm 9.3$ \\
\hline Sex & 12 \\
Male & 4 \\
Female & $1.3(1-4)$ \\
\hline No. of VT morphologies per patient* & $318 \pm 65$ \\
\hline VT cycle length (ms) & $26.0 \pm 9.3$ \\
\hline Scintigraphic LVEF (\%) & \\
\hline Echocardiographic dimensions & $91.8 \pm 9.7$ \\
LV end diastolic length (mm) & $56.4 \pm 7.9$ \\
LV end diastolic width (mm) & \\
\hline Extent of CAD ( $>$ 70 \% stenosis) & 6 \\
1 vessel & 5 \\
2 vessel & 5 \\
3 vessel & $8.7 \pm 5.1$ \\
\hline Time since infarction (years) & 10 \\
\hline Site of myocardial infarction & 6 \\
Anterior & \\
Inferior & \\
\hline
\end{tabular}

Table 1: Basic patient characteristics. All data shown as mean \pm std except ${ }^{*}$ as mean (range). 

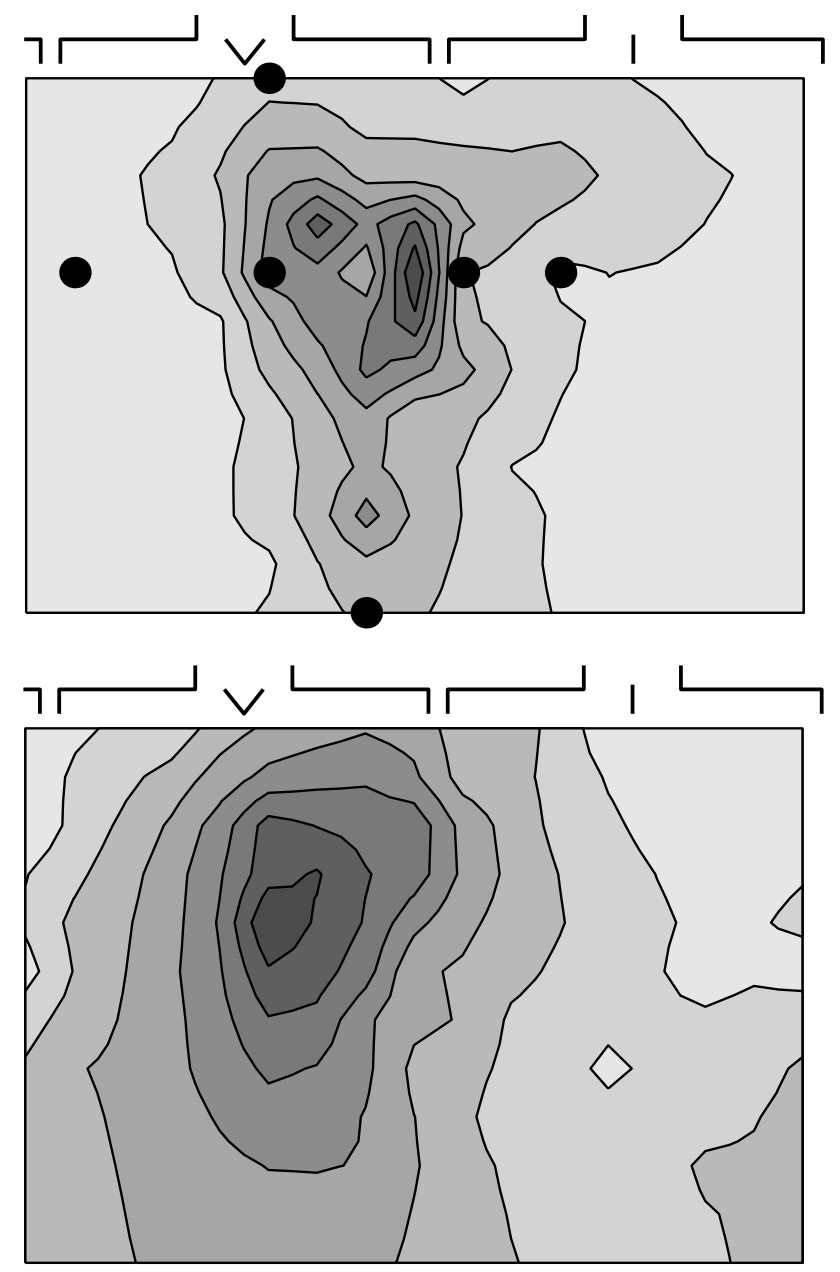

Fig. 1: Top panel: the energy distribution in the $50 \mathrm{~ms}$ after the J-point for a patient without late potentials in the standard signal averaged ECG. The approximate positions of the $X, Y$, and $Z$ electrodes are shown as dots. Lower panel: the mean energy distribution during the QRS in the same patient.

rather time consuming and only possible during catheterization. Another option would be to remove the preferential direction of the heart axis by high pass filtering. After filtering, the surface distribution consists of rapidly changing nearly dipolar patterns. Based on these physical considerations it was expected that high-pass filtered activation during the QRS complex may serve as a good estimation of the distance effect. Since detection of late potentials is done using high-pass filtered $\mathrm{X}, \mathrm{Y}$ and $\mathrm{Z}$ leads, using these same signals for the correction procedure seems very effective. To be able to compare maps from different patients the correction vector should have an amplitude that does not depend on the total energy in the QRS complex for that patient. This can be achieved by normalizing the correction factor.

\section{Results}

After correction for distance effects it was possible to link the signal averaged late potentials at the body surface to di-
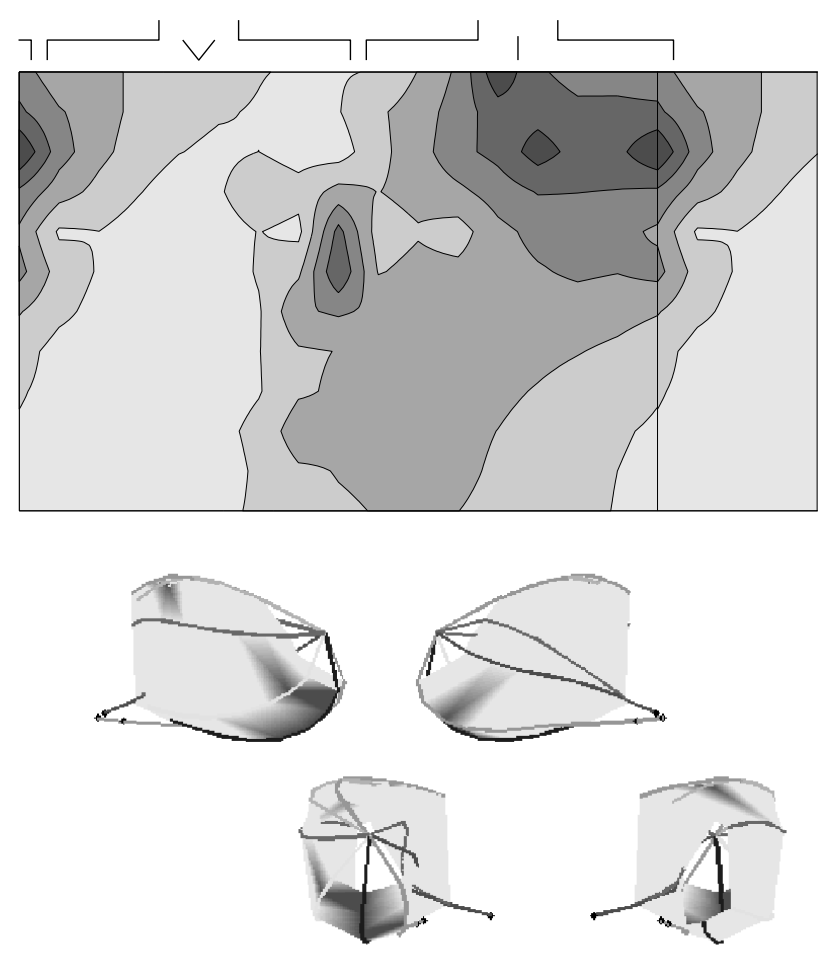

Fig. 2: Top panel: energy distribution in the same patient as in Fig. 1 after correction for the distance effect. Lower panel: the diastolic potentials as recorded with the basket catheter. Dark colors indicate electrodes with diastolic potentials. Note that the two areas with diastolic potentials correspond to the dark areas in the top panel.

astolic potentials in areas of the heart closest to the surface electrodes in 13 out of 16 patients.

In Fig. 1, a patient is shown who has no late potentials on the standard SAECG although this patient had documented VT. In the top panel the uncorrected energy of the late potentials is shown. In this panel also the approximate positions of the standard $\mathrm{X}, \mathrm{Y}$, and $\mathrm{Z}$ electrodes are shown. The standard SAECG procedure did not show late potentials probably because the area is relatively small and the electrodes are just outside this area. The lower panel shows the average energy during the QRS interval. Very clear is that, as expected, this is largest in the precordial region.

In the upper panel of Fig. 2, the corrected energy i.e. the energy after the QRS divided by the energy during the QRS is shown. Darker values correspond to higher energies. For clarity the left part of the map is repeated on the right. From this map we can see that the energy in the $\mathrm{V} 1-\mathrm{V} 3$ area is not a result of late potentials in local heart tissue, but that the maximum around V5 does reflect late potentials. Note that there is also an area high on the back of the patient that shows a high energy contribution. These two areas correspond to the two areas of the basket catheter (lower panel) that show late diastolic potentials during sinus rhythm, the dark areas in the lower panel. The basket is shown in four views, one from the front, one from the right, one from the back and one from the left. They are positioned under the map at positions that correspond to 

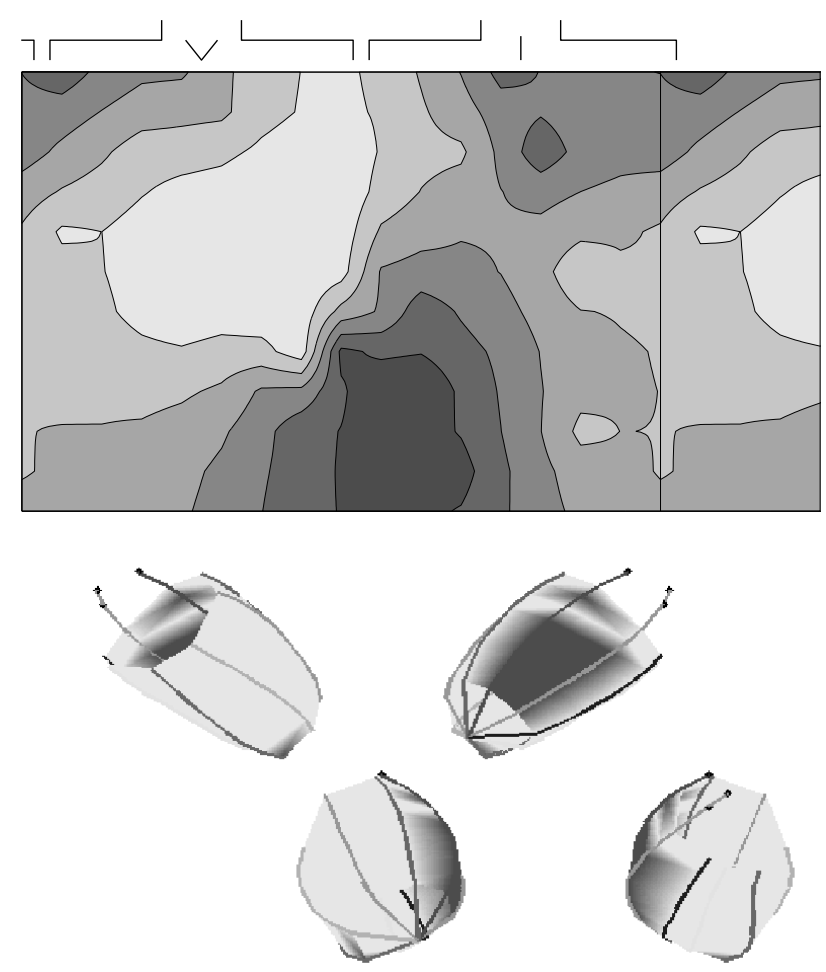

Fig. 3: A patient with late potentials. Layout as in Fig. 2.

these views.

In Fig. 3 data from a patient, who is positive for all three late potential criteria, are shown. In the literature various criteria for the detection of late potentials have been reported, depending among other things on the cutoff frequency used in the high pass filter $[2,3]$. In this study, late potentials were defined as a QRSD $>120 \mathrm{~ms}$, RMS40 $<23 \mu \mathrm{V}$, and LAS40 $>38 \mathrm{~ms}$ in accordance with commercially available equipment used in our hospital for traditional SAECG. Like in Fig. 2 the upper panel of Fig. 3 shows the the energy distribution over the body in the $50 \mathrm{~ms}$ after the $\mathbf{J}$ point, corrected for the distance. The lower panel shows the diastolic potentials as recorded by the basket catheter.

\section{Limitations of the study}

The basket catheter could not reach the entire endocardium, both due to the design of the catheter and to the fact that complete deployment was not always possible. Notably the apex and the basal part of the left ventricle were not completely covered. The fact that there is a large area of
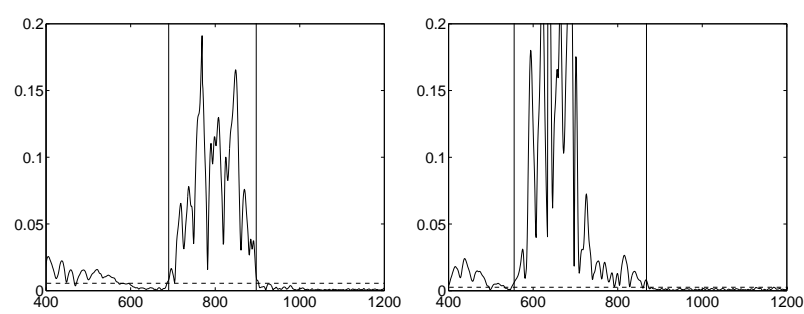

Fig. 4: SAECG plots for the two patients. The patient in the left diagram was considered to be without late potentials $(Q R S D=104 \mathrm{~ms}, \mathrm{RMS} 40=76.9 \mu \mathrm{V}, \mathrm{LAS} 40=10.5 \mathrm{~ms})$, whereas the patient in the right diagram has late potentials $(Q R S D=157.5 \mathrm{~ms}, \mathrm{RMS} 40=11.1 \mu \mathrm{V}, \mathrm{LAS} 40=69 \mathrm{~ms})$

late potential activation in the lower right frontal area in Fig. 3 while there seems to be only a couple of electrodes in the lower apical area with diastolic potentials, is probably due to this incomplete coverage of the apex by the catheter.

\section{Conclusions}

Late potential localization by SABSM is usually hindered by the fact that activation is most prominent in the precordial region due to the distance effect. The mean energy of the filtered QRS complex is mainly determined by this distance effect. We therefore propose to use the energy in the $50 \mathrm{~ms}$ interval after the J-point, divided by the energy in the QRS as a distribution that is sensitive to the origin of the late potentials.

\section{Acknowledgements}

This study was supported by grant AGN99.4098 of the Dutch technology foundation (STW).

\section{References}

[1] Simson, M. B. Use of signals in the terminal QRS complex to identify patients with ventricular tachycardia after myocardial infarction. Circulation, 64(2):235-242, 1981.

[2] Breithardt, G., Cain, M. E., El-Sherif, N., Flowers, N., Hombach, V., Janse, M., Simson, M. B., and Steinbeck, G. Standards for analysis of ventricular late potentials using high resolution or signal-averaged electrocardiography. A statement by a task force committee between the European Society of Cardiology, the American Heart Association and the American College of Cardiology. European Heart Journal, 12:473-480, 1991.

[3] Brachmann, J., Hilbel, T., Schweizer, M., and Kübler, W. Cardiac late potentials for diagnosis in heart failure. European Heart Journal, 14 Suppl. C:49-51, 1994. 